\title{
Hadron Spin Structure: Novel Effects from Transverse Single Spin Asymmetries ${ }^{1}$
}

\author{
U. D’Alesio \\ Dipartimento di Fisica, Università di Cagliari, and INFN, Sezione di Cagliari, C.P. 170, I-09042 \\ Monserrato (CA), Italy.
}

\begin{abstract}
Transverse single spin asymmetries can be a challenging tool in our understanding of the internal structure of hadrons. Some aspects and recent results are discussed.
\end{abstract}

Keywords: perturbative QCD, inclusive hadron production, spin effects

PACS: $12.38 . \mathrm{Bx}, 13.85 . \mathrm{Ni}, 13.88 .+\mathrm{e}$

Several experimental results clearly show that transverse single spin asymmetries (SSA) in high-energy hadronic collisions can be, in particular kinematics regions, very large. Two relevant examples are the transverse polarization, $P_{T}$, of $\Lambda$ produced in unpolarized hadron collisions and the left-right asymmetry, $A_{N}$, observed in $p^{\uparrow} p \rightarrow \pi X$ :

$$
P_{T}^{\Lambda}=\frac{d \sigma^{A B \rightarrow \Lambda^{\uparrow} X}-d \sigma^{A B \rightarrow \Lambda^{\downarrow} X}}{d \sigma^{A B \rightarrow \Lambda^{\uparrow} X}+d \sigma^{A B \rightarrow \Lambda^{\downarrow} X}} \quad A_{N}=\frac{d \sigma^{A^{\uparrow} B \rightarrow C X}-d \sigma^{A^{\downarrow} B \rightarrow C X}}{d \sigma^{A^{\uparrow} B \rightarrow C X}+d \sigma^{A^{\downarrow} B \rightarrow C X}}
$$

where $d \sigma$ stands for the corresponding invariant differential cross section and $\uparrow, \downarrow$ denote transverse polarization with respect to the hadron production plane. While these observables can reach in size values up to $30 \%-40 \%$, it is easy to see that in the usual collinear partonic kinematics, perturbative QCD (pQCD) predicts almost vanishing SSA. In fact at the partonic level single spin asymmetries are related to helicity flip amplitudes and to relative phases, both of which are absent in the perturbative, chirality conserving, leading order interactions of quarks and gluons. SSA are then sensitive to higher twist contributions, or non perturbative effects in the long distance physics, and are expected to vanish in the truly asymptotic, high-energy, large $Q^{2}$ (or $p_{T}$ ) regions.

Among the attempted explanations of $A_{N}$ and $P_{T}^{\Lambda}$ we consider here the approach based on pQCD dynamics, through a generalization of the factorization scheme: one starts from the leading twist, collinear configuration scheme and generalizes it with the inclusion of transverse motion of partons in distribution functions (PDF) and hadrons in fragmentation functions (FF). This leads, for the inclusive cross section for $A B \rightarrow C X$, to

$$
d \sigma^{A B \rightarrow C X}=\sum_{a, b, c, d} \hat{f}_{a / A}\left(x_{a}, \boldsymbol{k}_{\perp a}\right) \otimes \hat{f}_{b / B}\left(x_{b}, \boldsymbol{k}_{\perp b}\right) \otimes d \hat{\boldsymbol{\sigma}}^{a b \rightarrow c d}(\hat{s}, \hat{t}) \otimes \hat{D}_{C / c}\left(z, \boldsymbol{k}_{\perp C}\right),
$$

where $\otimes$ stands for convolution both on $x_{i}(z)$ and $\boldsymbol{k}_{\perp i}$.

As discussed in [1] the inclusion of intrinsic $\boldsymbol{k}_{\perp}$ effects could also play a relevant role in reducing the gap (in some cases very large) between the theoretical pQCD estimates and the experimental data for unpolarized inclusive particle production.

\footnotetext{
1 Talk delivered at the 6th Conference on "Quark Confinement and the Hadron Spectrum", September 21-25, 2004, Villasimius, Italy.
} 
For polarized processes the introduction of $k_{\perp}$ and spin dependences opens up the way to many possible spin effects; these can be summarized by the new functions:

$$
\begin{aligned}
\Delta^{N} f_{q / p^{\uparrow}} & \equiv \hat{f}_{q / p^{\uparrow}}\left(x, \boldsymbol{k}_{\perp}\right)-\hat{f}_{q / p^{\downarrow}}\left(x, \boldsymbol{k}_{\perp}\right)=\hat{f}_{q / p^{\uparrow}}\left(x, \boldsymbol{k}_{\perp}\right)-\hat{f}_{q / p^{\uparrow}}\left(x,-\boldsymbol{k}_{\perp}\right) \\
\Delta^{N} f_{q^{\uparrow} / p} & \equiv \hat{f}_{q^{\uparrow} / p}\left(x, \boldsymbol{k}_{\perp}\right)-\hat{f}_{q^{\downarrow} / p}\left(x, \boldsymbol{k}_{\perp}\right)=\hat{f}_{q^{\uparrow} / p}\left(x, \boldsymbol{k}_{\perp}\right)-\hat{f}_{q^{\uparrow} / p}\left(x,-\boldsymbol{k}_{\perp}\right) \\
\Delta^{N} D_{h / q^{\uparrow}} & \equiv \hat{D}_{h / q^{\uparrow}}\left(z, \boldsymbol{k}_{\perp}\right)-\hat{D}_{h / q^{\downarrow}}\left(z, \boldsymbol{k}_{\perp}\right)=\hat{D}_{h / q^{\uparrow}}\left(z, \boldsymbol{k}_{\perp}\right)-\hat{D}_{h / q^{\uparrow}}\left(z,-\boldsymbol{k}_{\perp}\right) \\
\Delta^{N} D_{h^{\uparrow} / q} & \equiv \hat{D}_{h^{\uparrow} / q}\left(z, \boldsymbol{k}_{\perp}\right)-\hat{D}_{h^{\downarrow} / q}\left(z, \boldsymbol{k}_{\perp}\right)=\hat{D}_{h^{\uparrow} / q}\left(z, \boldsymbol{k}_{\perp}\right)-\hat{D}_{h^{\uparrow} / q}\left(z,-\boldsymbol{k}_{\perp}\right) .
\end{aligned}
$$

The functions in Eq.s (3) and (5) are respectively the so-called Sivers [2] and Collins [3] functions; in Eq.s (4) and (6) we have the functions introduced by Boer and Mulders [4] and the so-called "polarizing" FF [4, 5]. Moreover the ones in Eq.s (4) and (5) are chiralodd, while the other two are chiral-even. All the above functions vanish when $k_{\perp}=0$, are naïvely $T$-odd and have a clear partonic interpretation. For instance, the Sivers mechanism corresponds to the azimuthal dependence (around the light-cone direction of the parent nucleon) of the number density of unpolarized partons inside a transversely polarized nucleon; the Collins mechanism corresponds to the azimuthal dependence (around the light-cone direction of the fragmenting parton) of the number density of unpolarized hadrons resulting from the fragmentation of a transversely polarized quark. Similar functions can be found in the literature with different notations $[4,6]$.

In principle both the Sivers and the Collins mechanisms could be responsible for the observed $A_{N}$ at E704 [7] (see [8]), while the polarizing FF in Eq. (6) could explain the measured transverse $\Lambda$ polarization in unpolarized hadron collisions [5].

Recent phenomenological studies [9] have shown how the detailed microscopic dynamics, with all the correct azimuthal angular dependences, produces a strong suppression of the transverse SSA arising from the Collins mechanism. The Sivers effect is not suppressed [1]. In Fig. 1 we show our latest results for $A_{N}$ both in terms of the Sivers effect alone (including only valence contributions) and with the Collins effect alone (maximizing and including all contributions). A complete study of single (and double) spin asymmetries within the helicity formalism including $\boldsymbol{k}_{\perp}$ effects is underway [10].

A few words on some theoretical developments are mandatory. In fact only in the last years the role played by the gauge link (Wilson line) entering the operator definition of these functions has been exploited. As a result we expect that whereas deep inelastic scattering (DIS) and Drell-Yan processes probe the same unpolarized PDF, they select Sivers PDF with opposite sign [11]. This poses obviously a question on universality.

As pointed out above, usually, more than one mechanism might in principle contribute to the same SSA. Therefore it is crucial to find proper ways to isolate each of them.

To this aim a combined experimental analysis of transverse SSA in Drell-Yan processes and semi-inclusive deep inelastic scattering (SIDIS) would be extremely useful. First data on azimuthal SSA arising from Sivers effect in SIDIS are now available [12] and more are coming from HERMES and COMPASS collaborations. On the theoretical side it has been shown how by proper suitable integration over the angular dependence of the lepton pair, a measurement of $A_{N}$ in polarized Drell-Yan processes would give a direct access to the (quark) Sivers function [13]. A tool to learn on possible Sivers effect from gluons could be through the process $p^{\uparrow} p \rightarrow D X$ at RHIC energies (heavy meson production), being dominated by the partonic subprocess $g g \rightarrow c \bar{c}$ [14]. 

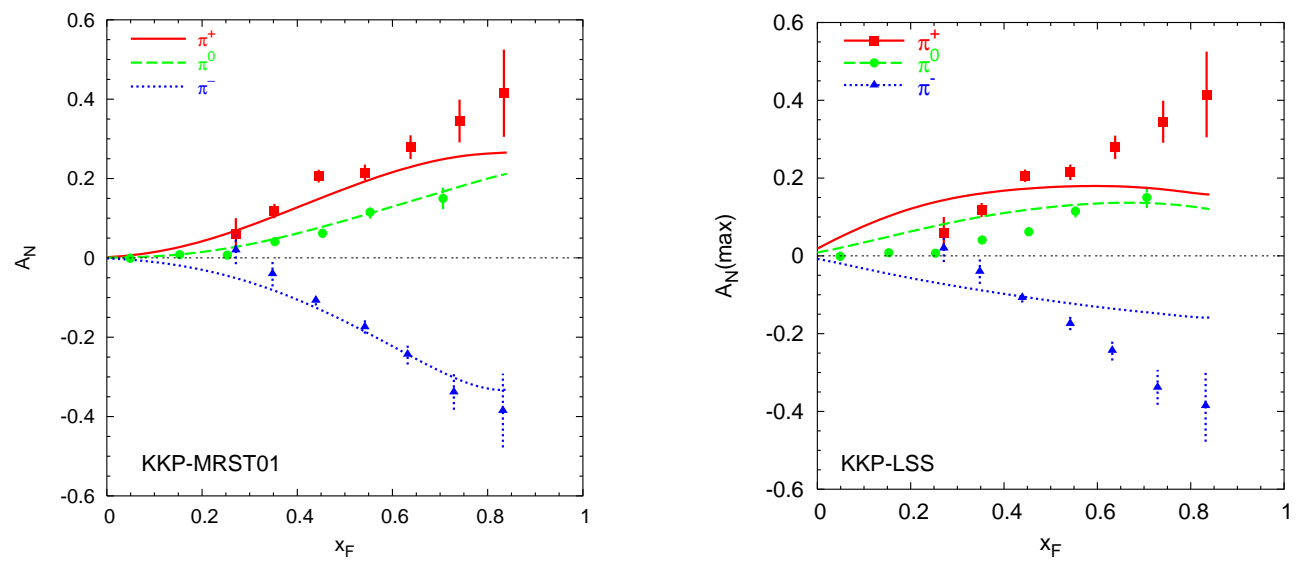

FIGURE 1. $A_{N}\left(p^{\uparrow} p \rightarrow \pi X\right)$ vs. $x_{F}$ at $p_{\mathrm{lab}}=200 \mathrm{GeV} / c$ and fixed $p_{T}=1.5 \mathrm{GeV} / c$. Curves are obtained with Sivers effect (left plot), and with Collins effect (right plot). See [1, 9] for details. Data are from [7].

Finally, Collins effect could play a crucial role in the extraction of the the still unknown, leading twist, transversity PDF, $h_{1}$, describing the quark transverse polarization inside a transversely polarized proton. Indeed due to its chiral oddness, the Collins function can be a partner of $h_{1}$ in the azimuthal SSA observed in $\ell p^{\uparrow} \rightarrow \ell^{\prime} \pi X$.

SSA offer a unique access to new information on hadron structure. This new class of spin and $\boldsymbol{k}_{\perp}$ dependent functions can give a much deeper insight of non perturbative and long-range physics. New data, soon available, will help in their interpretation. On the theoretical side, a better understanding of their fundamental properties, like universality, QCD evolution, factorizability, and classification would be extremely useful.

This brief overview is based on a series of papers in collaboration with M. Anselmino, M. Boglione, E. Leader, S. Melis and F. Murgia.

\section{REFERENCES}

1. U. D’Alesio and F. Murgia, Phys. Rev. D70, 074009 (2004).

2. D. Sivers, Phys. Rev. D41, 83 (1990); Phys. Rev. D43, 261 (1991).

3. J.C. Collins, Nucl. Phys. B396, 161 (1993).

4. P.J. Mulders, and R.D. Tangerman, Nucl. Phys. B461, 197 (1996), erratum ibid. B484, 538 (1997); D. Boer, and P.J. Mulders, Phys. Rev. D57, 5780 (1998); D. Boer, Phys. Rev. D60, 014012 (1999).

5. M. Anselmino, D. Boer, U. D’Alesio and F. Murgia, Phys. Rev. D63, 054029 (2001).

6. A. Bacchetta, U. D’Alesio, M. Diehl, C. Andy Miller, Phys. Rev. D70, 117504 (2004).

7. D.L. Adams et al., Phys. Lett. B264 (1991) 462; B261 201 (1991).

8. M. Anselmino, M. Boglione and F. Murgia, Phys. Lett. B362, 164 (1995); Phys. Rev. D60, 054027 (1999); M. Boglione and E. Leader, Phys. Rev. D61 114001 (2000).

9. M. Anselmino, M. Boglione, U. D’Alesio, E. Leader and F. Murgia, Phys. Rev. D71 014002 (2005).

10. M. Anselmino, M. Boglione, U. D’Alesio, E. Leader, S. Melis and F. Murgia, in preparation.

11. S.J. Brodsky, D.S. Hwang, and I. Schmidt, Phys. Lett. B530, 99 (2002); Nucl. Phys. B642, 344 (2002); J.C. Collins, Phys. Lett. B536, 43 (2002).

12. A. Airapetian et al. (HERMES Coll.) e-Print Archive: hep-ex/0408013.

13. M. Anselmino, U. D’Alesio, and F. Murgia, Phys. Rev. D67, 074010 (2003).

14. M. Anselmino, M. Boglione, U. D’Alesio, E. Leader, and F. Murgia, Phys. Rev. D70, 074025 (2004). 